\title{
Solar subsurface flows of active regions: flux emergence and flare activity
}

\author{
Rudolf Komm, Rachel Howe, Frank Hill, and Kiran Jain \\ National Solar Observatory \\ 950 N. Cherry Ave., Tucson, AZ 85719, USA \\ email: rkomm@nso.edu
}

\begin{abstract}
We study the temporal variation of subsurface flows associated with active regions within $16 \mathrm{Mm}$ of the solar surface. We have analyzed the subsurface flows of nearly 1000 active and quiet regions applying ring-diagram analysis to Global Oscillation Network Group (GONG) Dopplergram data. We find that newly emerging active regions are characterized by enhanced upflows and fast zonal flows in the near-surface layers, as expected for a flux tube rising from deeper layers of the convection zone. The subsurface flows associated with strong active regions are highly twisted, as indicated by their large vorticity and helicity values. The dipolar pattern exhibited by the zonal and meridional vorticity component leads to the interpretation that these subsurface flows resemble vortex rings, when measured on the spatial scales of the standard ringdiagram analysis.
\end{abstract}

Keywords. Sun: helioseismology, Sun: magnetic fields, Sun: flares

\section{Introduction}

We study the temporal variation of subsurface flows of active regions from the solar surface to a depth of $16 \mathrm{Mm}$. We have measured the subsurface flows of nearly 1000 active and quiet regions by analyzing Global Oscillation Network Group (GONG) Dopplergram data with the ring-diagram technique. As part of an on-going study, we are focusing on (1) emerging active regions and (2) the twist of subsurface flows and its role in the flare activity of active regions. In previous studies (Komm et al. 2009), we have found that the vertical velocity component is a good indicator of temporal changes in magnetic flux. Here, we focus on emerging active regions and the zonal (east-west) velocity component in addition to the vertical one.

The other subject of interest here is the vorticity of subsurface flows. In previous studies (Komm et al. 2011; Reinard et al. 2010), we have shown that the flare activity of solar active regions is intrinsically linked with the vorticity of solar subsurface flows on spatial scales comparable to the size of active regions and on temporal scales from days to the lifetime of active regions. Here, we have a closer look at the vortical structure of subsurface flows of strong active regions.

\section{Data and Method}

We determine the horizontal components of solar subsurface flows with a ring-diagram analysis using the dense-pack technique (Haber et al. 2002). The full-disk Doppler images obtained with GONG are divided into 189 overlapping regions with centers spaced by $7.5^{\circ}$ ranging from $\pm 52.5^{\circ}$ in latitude and central meridian distance $(\mathrm{CMD})$. Each region is 

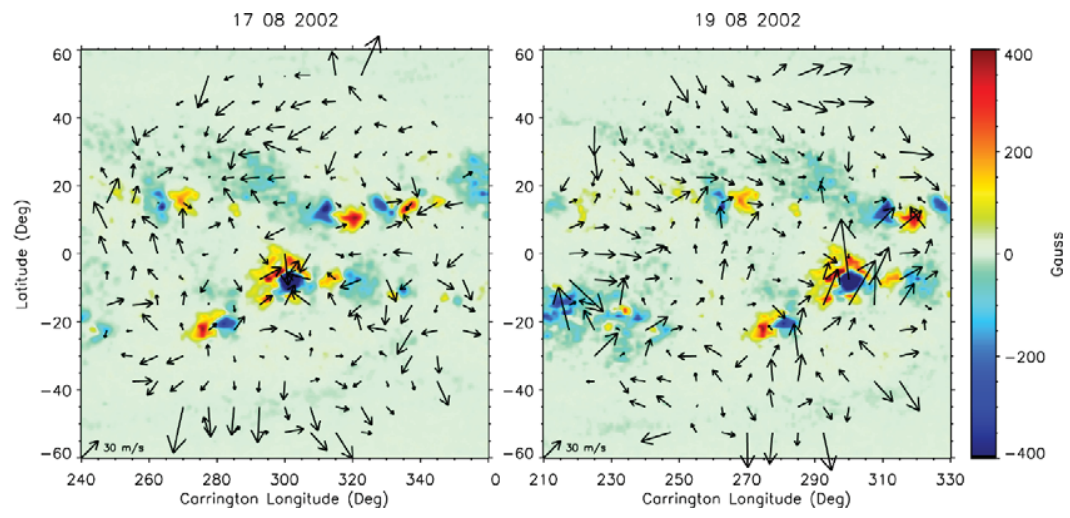

Figure 1. Daily horizontal-flow maps at a depth of $7 \mathrm{Mm}$ with AR 10069 moving across the Sun. Arrows indicate flows; the background shows the magnetic activity (from a synoptic map).

apodized with a circular function reducing the effective diameter to $15^{\circ}$ before calculating three-dimensional power spectra. The data are analyzed in "days" of 1664 minutes. Each of these regions is tracked throughout the sequence of images using the appropriate surface rate. For each dense-pack day, we derive maps of horizontal velocities at 189 locations in latitude and CMD for 16 depths from 0.6 to $16 \mathrm{Mm}$. Figure 1 shows two examples. The residual vertical velocity is estimated from the divergence of the horizontal flows assuming mass conservation (Komm 2007). To study the twist of the subsurface flows, we calculate the vorticity, defined as the curl of the velocity vector, and the kinetic helicity density, defined as the scalar product of the velocity and vorticity vector (Moffatt \& Tsinober 1992).

As a measure of solar activity, we use Michelson Doppler Imager (MDI) magnetograms. We determine the change in unsigned magnetic flux during the disk passage of each active region by averaging 96-min MDI magnetograms in time over the length of a ring-diagram day and bin them to the dense-pack spatial grid. To study the emergence of active regions, we sort the data set by the average change in magnetic flux during the disk passage of each region. We then select 83 regions (10\% of all regions) with the largest increase in activity starting from small activity values. For each emerging region, we determine the day of emergence as the first day with a flux increase of $10 \%$ and center each time series on this day.

\section{Results}

Figure 2 shows the average zonal and vertical velocity component associated with emerging active regions. For the zonal velocity component, a band of faster-than-average flow values moves from a depth of $16 \mathrm{Mm}$ to the surface within two days of emergence. This agrees with the fact known from surface and helioseismic observations that active regions rotate faster than the ambient fluid. For the vertical velocity component, upflows increase with increasing flux at depths greater than $10 \mathrm{Mm}$. Between about 4 and $10 \mathrm{Mm}$, weak upflows change to downflows two days after flux emergence. This means that at the end of the time series, the average depth variation has been established with upflows in deeper layers and downflows in shallow ones. The time scale of two days coincides with the active phase of growth of newly emerging active regions. 

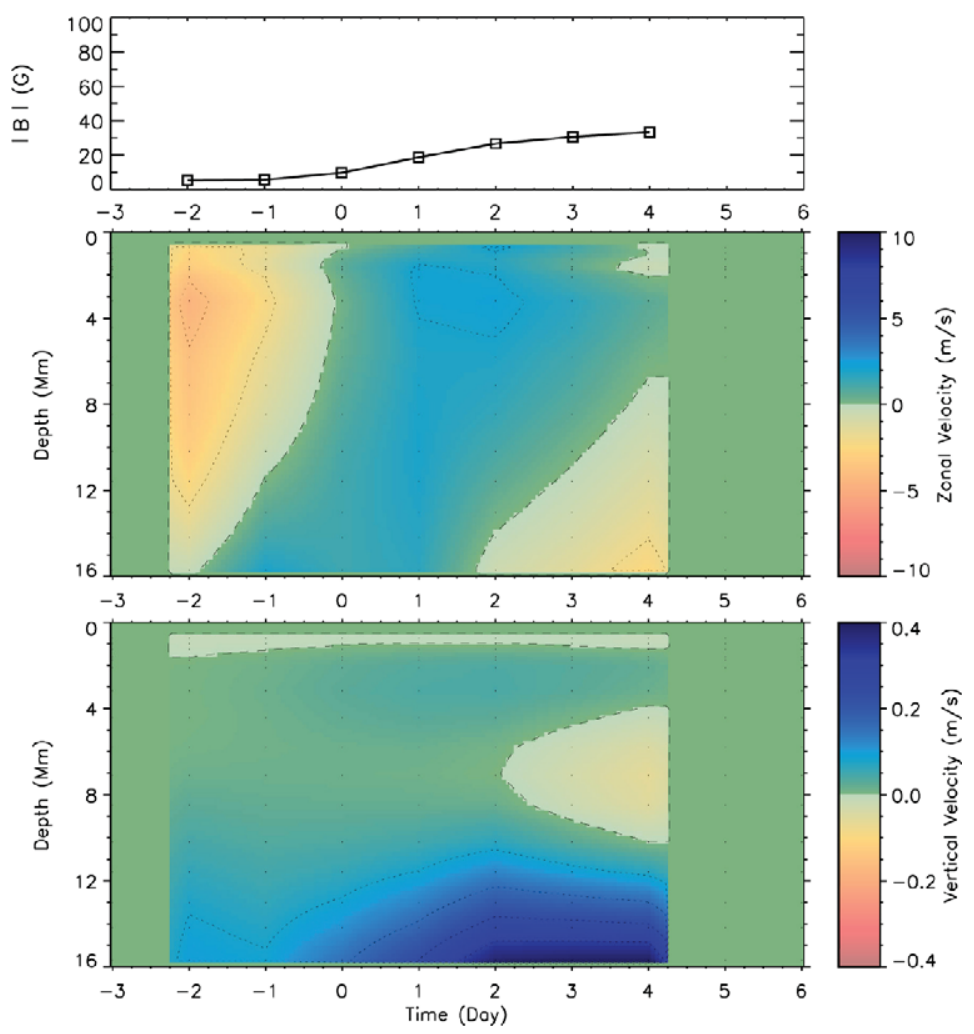

Figure 2. Emerging active regions and their subsurface flows. Top: The residual zonal velocity averaged over 83 emerging regions as a function of time and depth. Bottom: The corresponding residual vertical velocity. Positive (negative) values indicate faster (slower) zonal flows and upflows (downflows) in the vertical component. The mean value has been subtracted at every depth for the zonal flow. The line drawing indicates the unsigned magnetic flux.

Figure 3 shows, as example, the average kinetic helicity density of a strong flareproductive active region (10069). Strong active regions show a "dipolar" pattern in the kinetic helicity density that changes sign with depth. This pattern originates in the zonal and meridional vorticity component of the subsurface flows. The zonal vorticity shows this pattern in the north-south direction, while the meridional vorticity component shows a similar pattern aligned in the east-west direction, when measured on dense-pack scales. We interpret this pattern as representing two vortex rings stacked on top of each other, as shown in the schematic diagram. There might exist a third vortex ring in the shallow layers within $2 \mathrm{Mm}$ of the solar surface.

\section{Summary}

We study subsurface flows of active regions within $16 \mathrm{Mm}$ of the solar surface. We find that newly emerging active regions are characterized by enhanced upflows and fast zonal flows in the near-surface layers, as expected for a flux tube rising from deeper layers of the convection zone. After active regions are formed, downflows are established within two days of emergence in shallow layers between about 4 and $10 \mathrm{Mm}$. A detailed study will appear in the near future (Komm et al. 2011). 

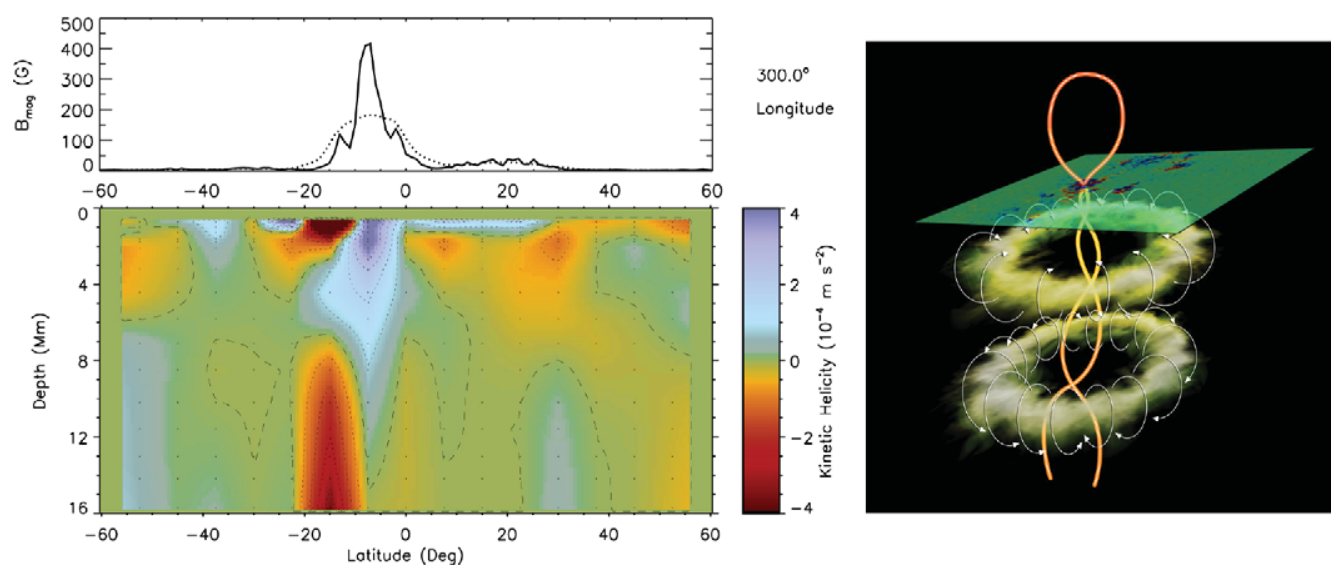

Figure 3. Left: The kinetic helicity density as a function of latitude and depth for AR 10069 (at $300^{\circ}$ Carrington longitude). The peak of the unsigned magnetic flux in the line drawing indicates the location of the active region. Right: Schematic of the twisted subsurface flows associated with strong active regions. On dense-pack scales, these flows resemble stacked vortex rings centered on a flux tube (Courtesy: P. Marenfeld).

The subsurface flows associated with strong active regions are highly twisted, as indicated by the large vorticity and helicity values. The dipolar pattern exhibited by these quantities when measured on dense-pack scales leads to the interpretation that subsurface flows associated with strong active regions resemble vortex rings (Shariff \& Leonard 1992). Previous studies have shown that flare-productive active regions have large values of magnetic activity and subsurface vorticity or helicity (Komm \& Hill 2009). Subsurface vorticity and helicity thus provide information about the flare productivity of active regions. We plan to investigate the temporal variation of the vorticity pattern and its relation to the evolution of active regions in the near future.

\section{Acknowledgements}

This work utilizes data obtained by the Global Oscillation Network Group (GONG) program, managed by the National Solar Observatory, which is operated by the Association of Universities for Research in Astronomy (AURA), Inc. under a cooperative agreement with the National Science Foundation. The data were acquired by instruments operated by the Big Bear Solar Observatory, High Altitude Observatory, Learmonth Solar Observatory, Udaipur Solar Observatory, Instituto de Astrofísica de Canarias, and Cerro Tololo Interamerican Observatory. SOHO is a mission of international cooperation between ESA and NASA. This work was supported by NASA grant NNG08EI54I to the National Solar Observatory.

\section{References}

Haber D. A., Hindman, B. W., Toomre, J., Bogart, R. S., Larsen, R. M., \& Hill, F. 2002, ApJ, 570,885

Komm, R. 2007, Astron. Nachr., 328, 269

Komm, R., Howe, R., \& Hill, F. 2011, Solar Phys., 268, 407

Komm, R., Ferguson, R., Hill, F., Barnes, G., \& Leka K. D. 2011, Solar Phys., 268, 389 
Komm, R. \& Hill, F. 2009, J Geophys. Res., A06105

Komm, R., Howe, R., \& Hill, F. 2009, Solar Phys., 258, 13

Moffatt, H. K. \& Tsinober, A. 1992, Ann. Rev. Fluid Mech., 24, 281

Reinard, A. A., Henthorn, J., Komm, R., \& Hill, F. 2010, Astrophys. J. Lett., 710, L121

Shariff, K. \& Leonard, A. 1992, Ann. Rev. Fluid Mech., 24, 235 